\title{
Correction to: Letter to the Editor-Recent advances in Lynch syndrome
}

\author{
Pål Møller ${ }^{1}$ (1) - Julian Sampson ${ }^{2} \cdot$ Mev Dominguez-Valentin ${ }^{1} \cdot$ John Burn $^{3} \cdot$ Lone Sunde $^{4,5} \cdot$ Gabriela Möslein $^{6}$. \\ Jukka-Pekka Mecklin ${ }^{7} \cdot$ Toni Seppälä8
}

Published online: 1 April 2021

(c) Springer Nature B.V. 2021

\section{Correction to: Familial Cancer \\ https://doi.org/10.1007/s10689-020-00200-6}

Unfortunately, the original article has been published incorrectly with article title "Recent advances in Lynch syndrome, the correct title is "Letter to the Editor-Recent advances in Lynch syndrome".

The Original article has been corrected.

Publisher's Note Springer Nature remains neutral with regard to jurisdictional claims in published maps and institutional affiliations.

The original article can be found online at https://doi.org/10.1007/ s10689-020-00200-6.

Pål Møller

moller.pal@gmail.com

1 Department of Tumor Biology, Institute of Cancer Research, The Norwegian Radium Hospital, Oslo University Hospital, Oslo, Norway

2 Division of Cancer and Genetics, Institute of Medical Genetics, Cardiff University School of Medicine, Cardiff, UK

3 Faculty of Medical Sciences, Newcastle University, Newcastle upon Tyne, UK

4 Department of Clinical Genetics, Aalborg University Hospital, Aalborg, Denmark

5 Department of Biomedicine, Aarhus University, Aarhus, Denmark

6 Center for Hereditary Tumors, University Witten/ Herdecke, Ev. Bethesda Khs zu Duisburg, Witten, Germany

7 Department of Surgery, Central Finland Health Care District, and Sport and Health Sciences, University of Jyväskylä, Jyväskylä, Finland

8 Department of Surgery, Helsinki University Hospital, Helsinki, Finland 